\title{
Author Index Vol. 70, 1995
}

Abe,K,K. 28 Adams, C.E. 49 Aggarwal, S.K. 123,385 Aguilar,D. 402 Akikusa,B. 376

Alikasifoglu, M. 388 Amagasaki, Y. 455 Amici,G. 506 Amoedo, M.L. 262 Anarat,A. 410

Anastasio, P. 421 Andersson, A. 62 Andres, A. 35 Andújar, M. 402 Aneiros, J. 402 Angelini,D. 193 Antonelli,A. 193 Anzai, N. 376 Aparicio, M. 287 Arai,M. 374 Arakawa,K. 28 Arbeiter, K. 255 Arici,C. 180 Arnadottir, M. 62 Atkins, R.C. 391 Avasthi,P.S. 296

Bäck,S.-E. 430 Baglin,A. 267 Bailey, R.R. 104 Bandini,S. 193 Bang, B.K. 372, 378, 397,499 Bariety,J. 249 Barsotti,G. 193,271 Basile,C. 133 Battini,G. 106 Beauchet,A. 267 Bedani,P.L. 301 Bennett, R. 387 Bererhi,L. 197 Bernard, A. 438 Bernardo, J.F. 235 Bertolini,S. 500 Bhandari,M. 269 Blanchetier, V. 287 Blöhmer,A. 425 Bommel, E.F.H. van 185 Bonilla,F. 139 Borghi,M. 131 Borrás, M. 262 Borrellas,X. 114 Bouchet,J.L. 287 Bournerias, F. 197 Bouvy,N.D. 185 Branch, R.A. 235 Breckenridge, C. 155 Breuning, M.H. 388 Brown, P. 127 Braining, HA. 185 Bulatovic, G 112 Bustos,M. 402

Campistol, J.M. 389 Canbolat,O. 380 Carroll, HJ. 143 Carozzi,S. 106 Casl,M.T. 112

Castagnone, D. 314 Chacon, P. 264 Chagnac, A. 207 Chang, C.-C. 91 Chang, Y.S. 372,397

Chen,C.-F. 83,91 Chen,G 121 Chen,H.-C. 223 Chen,Y.-M. 83 Cheong,H.I. 319 Choi,E.H. 319 Choi,K.B. 378,499 Choi,Y. 319 Choukroun,G. 11, 18 Chuet,C. 136 Chun,H.K. 499 Cirillo,M. 421 Cohen, E.P. 217 Combe, C. 287 Consoli,N. 267 Coremans, P. 494 Corvinelli, M. 421 Cruz, A. 508 Cukuranovic, R. 504 Cupisti,A. 193

Dani,L. 193 Darlametsos, I. 249 Dash,S.C. 123,385 De Felice, C. 115 Del Vecchio Blanco, C. 421 De Marchi, M. 106 Demircin, G. 260 De Paoli Vitali, E. 301 DeSanto,N.G. 421 Deulofeu,R. 389 Dioudis, C. 25 Dirks, B. 110 Dojcinov, D. 504

Dorairajan, L.N. 385 Dorhout Mees, E.J. 1 Driessen, M. 334 Drüeke,T.B. 197 Durak,I. 380 Eckardt, K.-U. 197 Eguchi, K. 42 Eisenberg,B. 296 Eke,F.U. 132 Eke,N. 132 Elli,A. 314 Endo,Y. 348,497 Enriquez, R. 139 Es,P.N.van 137 Estepa,M.R. 307

Fernandez, E. 262 Fernandez Lucas, M. 282 Ferrer-Prevosti, E. 114 Finielz,P. 136 Fiorini,F. 500 Fleming, S.J. 49 Fogazzi, G.B. 131 Fournier, A. 287 Frauscher, G. 255 Freed, B. 387

Fujitsuka,N. 382 Fukagawa, M. 242 Fukui,M. 369 Fushimi, T. 455

Gafter,U. 202,207 Gallego,N. 307 Gámez,C. 307 Garcia del Moral, R. 402 Gault,M.H. 155 Ghoneim, M. 478 Ghosh, A.K. 266 Gibbons, N. 229 Gilli,P. 301 Giordano, F. 106 Giovannetti, S. 193 Gkikas, G. 247 Gmehlin,U. 110 Goguel,A. 267 Gökhun,I.H. 380 Gomez-Ainsua, C. 508 Gómez-Morales, M. 402 Gönlüß̧en,G. 410 Gonzalez, E. 139 Gonzalez-Gay, MA. 270 GonzalezLorenzo, J. 438

Gotloib,L. 466 Goto,T. 324 Grekas,D. 25 Grenda,R. 77 Groppoli,T. 42 Grünfeld, J.-P. 11,18 Guh,J.-Y. 223 Guiserix, J. 136 Guleria,S. 123,385 Gündogdu, M. 141 Guo,M. 121 Gupta, A. 269 Gurgiolo,P. 387

Ha,I.S. 319 Hamasaki, K. 374 Hamed,S. 478 Hanamoto, N. 171 Hänel,H. 425 Hara,M. 348 Harnett,J. 155 Harris, D.C.H. 340 Hasegawa, A. 455 Hayakawa, K. 115 Hayashi,H. 348 Heidemann, H.T. 425 Hemmi,H. 455 Hernandez, E. 35 Hillis, G. 127 Hinoshita,F. 348,497 Hirai,H. 374 Hirai,K. 100,449 Hirasawa, Y. 125 Hiratsuka, T. 28 Hironaka,T. 100,449 Hirsh,J. 
207 Hökhun,I.H. 380 Horita,S. 116 Hornych,A. 249 Hosoya,T. 359 Hotz,P. 438 Houbouyan, L. 267 Houhou, S. 197 Huang, T.-F. 83 Hultberg,B. 62 Hwang, S.-J. 223 Hyodo, T. 171

Ichida,K. 359 lino, A. 171 Inoue, A. 455 Inoue, M. 460 Inselmann, G. 425

510

Ip,M. 367

Ippolito, G. 180

Ishigami, M. 455

Ishikawa, S. 497

Isoda,K. 502

Italian Multicenter Study on Nosocomial and Occupational Risk of Infections in Dialysis 180

Ito, K. 68, 376

Itoh,K. 359

Jagger,J. 180 Januszewicz, P. 77 Joh,K. 359 Jung,K. 370 Jung,M. 370

Kanahara,K. 129 Kanbayashi, H. 348 Kang,D.H. 378 Kasai,S. 324 Kashiwagi,H. 382

Kavutcu,M. 380 Kawaguchi, Y. 348 Kawai,T. 348 Kerr,P.G. 391 Khazanchi, R.K. 385 Kher,V. 269 Kim,H.-J. 143 Kim,T. 324 Kim,Y. 42

Kim,Y.S. 372,378,397,499 Kishimoto,T. 324 Kitamura, N. 443 Ko,F.-N. 91 Kobayashi, Y. 443 Koh,Y.B. 397 Kohsaka,T. 416 Koide,H. 329 Kojima, T. 443 Komatsu, Y. 68 Koomans, H.A. 1 Korzets,A. 202,207 Kottny,W. 425 Kumagai, M. 242 Kumar, A. 269 Kumar, P. 269 Kuomi,T. 171 Kurkus,J. 430 Kurokawa,K. 242 Kurtz, A. 197 Kushihata,S. 353 Kushnier, R. 466 Lacueva, J. 139 Lai,K.N. 367 Lai,Y.-H. 223,486 Lambrecht, G.L.Y. 494 Lan,H.Y. 391 Lauwerys, R. 438 Lawton, C.A. 217 Lee,HJ. 319 Lee,S.H. 378,499 Lee,Y.-J. 486 Leeuw, P.W. de 137 León,M. 35 Levi,J. 207 Liaño,F. 282 Liew,C.T. 367 Lin, R.-H. 91 Lin,S.-R. 486 Ljutic,D. 130 Llobregat, R. 139 Loguercio, C. 421 Longerich, L.L. 155 Lopez, J.R. 270 Lubec,B. 255

Macken,P. 120 MacLeod, A. 127 Mac-Moune Lai, F. 367 Maduell,F. 135 Malbrain, M.L.N.G 494 Mamet,R. 202 Marcén, R. 282, 307 Martin, M. 264 Maruyama, N. 502 Marx,Th. 110 Masturzo, P. 500 Matsuda,J. 324 Matsumoto, K. 118 Matsumoto, M. 55 Matsumura, O. 502 Matsuya, S. 374 Mauer,S.M. 42 Medina-Cano, M.T. 402 Meroni, M. 106 Michinaga, I. 28 Mihara,S. 171 Mij,M. 500 Mimura, T. 374 Mitarai,T. 502 Mittal,R. 123 Mittal,S.K. 123 Miyagawa,II. 171 Miyazaki, S. 125 Mizokuchi, M. 369 Mizuiri,S. 455 Mondon, J.-M. 136 Montes,A. 402 Montoliu, J. 262 Moon,S.S. 430 Moorthy,A.V. 120 Mora, A. 139 Morel, D. 287 Mori, A. 382 Morigga, M. 131 Morino,N. 374 Moriya, T. 72

Morphake,P. 249 Moulder, J.E. 217 Moulonguet-Doleris, L. 267 Moustafa,F. 478 Moutabarrik, A. 55 Murakami, S. 235 Murata,G.H. 296

Nagasawa, R. 502 Nagata, M. 68 Naito,S. 28 Nakahama,H. 211 Nakajima, Y. 100,449

Nakamoto, T. 374 Nakamura, A. 416 Nakamura,Y. 100,449 Nakanishi, I. 55 Nakanishi,T. 211 Nakayama, M. 460 Nankivell, BJ. 340 Navarro,J.F. 282 Nellessen,U. 425 Nihei,H. 116 NikolicPaterson, D.J. 391 Nilsson-Ehle, P. 430 Nishihara, F. 211 Nishimura, Y. 460 Nitta,K. 116 Nojima, Y. 374 Nomoto,Y. 42 Noyan, A. 410

O’Bryan,T. 266 Oda,H. 353 Ode,T. 171 Ogahara,S. 28 Ogawa, M. 376 Ogawa, T. 353

Ogura,M. 348 Ogura,Y. 348,497 Oh, M.S. 143 Oh,Y.S. 499 Ohara,T. 455 Ohmuro,H. 329

Ohno,S. 72 Ohto,M. 376 Okabe,H. 359 Okada,K. 122 Okada,M. 125 Okutani,M. 171 Oner, A. 260 Ono,A. 443 Ono,K. 171 Orlic,P. 112 Orofïno,L. 307 Ortuño, J. 282, 307 Ota,K. 116

Otero,J. de 114 Oura,H. 382 O’Valle,F. 402

Ozasa,H. 116 Öztürk,S. 380 
Pais,B. 262 Pan,C.Q. 229

Papanikolaou, N. 249 Papoulidou, F. 25 Park,I.S. 372 Parra,E. 389 Parthe,V. 270 Peters, D.J.M. 388 Petrosillo, N. 180 Piera,L.L. 114,264 Pietrucci, A. 106 Polat,S. 410 Potaux,L. 287 Praga, M. 35 Précigout, V. de 287 Preuss, R. 334 Prinseau, J. 267 Purchase, L. 155 Puro,V. 180 Quarto di Palo, F. 314

Rai,P. 269 Ramirez, C. 402 Rault,R•M. 387 Renieri, A. 106 Rivolta,R. 314 Robles, N.R. 508 Rodicio, J.L. 35 Rojas,B. 270 Ruilope, L.M. 35 Rumboldt,Z. 130

Sabater,J. 307 Sabljar-Matovmovic, M. 112 Sabra,R. 235 Saeki,A. 359 Sagar, S. 229 Sagripanti, A. 271 Saji,M. 359 Saka,S. 329 Sakai,H. 42 Sakai,O. 348,359 Salvadori,M. 193 San,A. 141 Sasai-Takedatsu, M. 443 Sato,T. 460 Savic, V. 504 Saxena,S. 123,385 Schoenfeld, N. 202 Schrey,G. 137 Segarra, A. 264 Sessa,A. 106 Seung,K,B. 378,499 Seya,T. 55 Shand,B.I. 104 Sharma,R.K. 269

Shasha, S.M. 21 Sheu,J.-R. 83 Shimatake, H. 455 Shin,S.-J. 486 Shio,H. 171 Shirai,D. 211 Shirato, I. 329, 369 Shkolnik, T. 21 Shostak,A. 466 Shurtz-Swirski, R. 21 Siles,E. 438 Singhal,P.C. 229 Sinha,S. 385 So,KX. 185 Sobh,M. 478 Soffritti,S. 301 Soy,D. 389 Spitali,L. 421 Stefanovic, V. 504 Steffes, M.W. 42 Stewart, K. 127 Stoltz,J.F. 267 Sugimura, K. 324 Sugita,M. 211 Sungur,C. 388 Suzuki, D. 42 Suzuki,|T. 416 Suzuki,|Y. 329,348

Tabe,A. 359 Taguchi, T. 353 Takahashi, S. 122 Takahashi,Y. 329

Takamitsu, Y. 211 Takemoto,Y. 324 Taketani,S. 443 Tam,J.S.L. 367 Tan,M.-S. 486 Tanaka, T. 115 Tanaka,Y. 211 Tanegashima, M. 455 Tang,Z. 369 Tarelli,L.T. 106 Tashiro,K. 329 Tazzari, S. 106 Tekin,S.B. 141 Teng,C.-M. 91 Teng,H.T. 137 Terentieva, E. 115 Teruel, J.L. 282, 307 Tesch,G.H. 391 Thibodeaux, L.C. 265 Tiwari,S.C. 123,385 Tokieda,K. 28 Tomino,Y. 329,369 Tomita,K. 460 Tonbul,Z. 141 Tourkantonis, A. 25 Trujillano, G. 438 Tsai,J.-C. 223 Tsai,J.-H. 223,486 Tsai,T.-J. 83,91 Tsipas,G. 249 Tsuru,N. 28 Tuncer, í. 410 Tzamaloukas, A.H. 296 Ueda,M. 171 Ueda,S. 376 Ulrich,W. 255 Ulutepe,S. 380 Ureña,P. 197 Urti, D.A. 193 Valderrama, E. 229 Valente,U. 106 Valentine, E. 387 Valkouma,D. 25 Varet,B. 11,18 Vendramin, G. 131 Verbist,L. 494 Verhaegen, H. 494 Villafruela, J.J. 282 Vincent, H.H. 185 Virga,G. 506 Vreugdenhil, G. 137

Waisbrut,V. 466 Walker, RJ. 104 Wang,L.N. 369 Wardle,E,N. 151 Watanabe,T. 242 Wedel,T. 334 Weimar, W. 185 Weinstein, T. 207 Westhuyzen, J. 49 Wetterling, T. 334 Willingham, W. 387

Yagame, M. 42 Yajima, Y. 72 Yamada,A. 348 Yamagami, S. 324 Yamaguchi,Y. 68,116 Yamakido, M. 353 Yamamoto, M. 449 Yamamoto,T. 100,449 Yamato,H. 242 Yang, C.W. 372, 378, 397,499 Yazaki,Y. 374 Yen,C.-J. 83 Yi,H. 242

Yilmaz Selçuk, N. 141 Yokoyama,K. 348 Yokozawa, T. 382 Yoon,S,A. 397 Yoon,Y.S. 372,397 Yorioka,N. 129,353 Yoshikawa, H. 455 Yoshimoto, M. 324 Yoshino,Y. 171 Yumura,W. 116 Zager,P.G. 296 Zaid,D. 55 Zawadzki, J. 77 Zevin,D. 207 Zhang,D. 121 Zhu,D. 42 Zietse,R. 185

512

Author Index 